\title{
Analysis of Nonlinear Aeroelastic and Structural Characteristics of Wing / Multi External Storage System with High Aspect Ratio
}

\author{
Fengnan SUN ${ }^{1}$, Yan ZHU, Qiyou CHENG, Siwen WANG and Xuyang LIN \\ China Helicopter Research and Development Institute, Jingdezhen 333001, China
}

\begin{abstract}
Aiming at the problem of large deformation geometric nonlinearity of high aspect ratio wing/multi external storage system affected by aerodynamic, multi external storage and gravity loads, the nonlinear aeroelastic motion equation of wing is established by using medium deformation beam theory and quasi steady aerodynamic model, and the effects of geometric nonlinear deformation of high aspect ratio wing/multi external storage system on structural modal frequency Influence of vibration mode and flutter characteristics. The analysis results show that the influence of geometric nonlinear effect is mainly reflected in the first-order mode of lead-lag, and its flutter mechanism is flexural torsional coupling flutter dominated by the first-order mode of lead-lag. The random wing/multi plug-in system changes from vertical downward bending state to horizontal state, the influence of geometric nonlinear effect is weakened, and the amplitude of the first-order mode shape of lead-lag increases, Moreover, the variation trend of the first-order lead-lag and torsional modal frequencies slows down, which correspondingly reduces the variation amplitude of flutter velocity at the corresponding angle of attack, which shows that it has a certain positive effect on the flutter velocity by slowing down the geometric nonlinearity of the wing/multi plug-in system.
\end{abstract}

Keywords. Geometric nonlinearity, aeroelastic response, structural mode, flutter velocity.

\section{Introduction}

In recent years, high aspect ratio wing/multi propeller coupling system is one of the important development directions of new unmanned aerial vehicles. Typical representatives include NASA electric tilt wing GL-10 and Helios "Helios" UAV [1-2]. Multiple propellers are distributed on the wing, which is equivalent to multiple external stores, resulting in large aspect ratio, so that the geometric nonlinear factors in the deformation process can not be ignored, and serious structural elastic deformation will change the dynamic characteristics of the wing structure and then affect its flutter characteristics [3-5]. Therefore, in-depth study on the influence of geometric nonlinear deformation of wing / multi external storage system on structural mode, and revealing

\footnotetext{
${ }^{1}$ Corresponding Author, Fengnan SUN, Helicopter Research and Development Center, Airport, Tianjin, China; E-mail: sfn18322883097@163.com.
} 
the variation law of wing aeroelastic characteristics and structural dynamic characteristics is helpful to optimize its aeroelastic design.

The geometric nonlinear effect caused by the large deformation of the wing / multi external storage system structure with high aspect ratio will cause the change of its own stiffness characteristics, which will seriously affect the dynamic characteristics of the wing [6-7]. Scholars at home and abroad have done a lot of research on this problem. Librescu and Song [8] studied the dynamic response and free vibration characteristics of the wing / external storage system. Mayuresh and Dowell [9] studied the nonlinear aeroelasticity of the wing with high aspect ratio by combining theory and experiment. An Xiaomin and Xu Min [10] solved the geometric nonlinear aeroelasticity problem for CFD/CSD coupling. Based on Co-rotational theory, A nonlinear aeroelastic solution method under large geometric deformation is developed, Based on the co rotational theory, Wang Wei [11] established the geometric nonlinear structural model of the wing of high-altitude long endurance solar UAV, studied the static aeroelastic stability of the wing considering the geometric large deformation effect, and Xie Chang-chuan [12-13] and others analyzed the flutter characteristics of the wing with high aspect ratio based on the trim linearization method using NASTRAN software. Hu Zhi-yong [14] and others analyzed the nonlinear aeroelastic problem of the wing of a solar UAV by combining the geometric nonlinear structural finite element and nonlinear aerodynamic calculation method.

In this paper, the coupled aeroelastic motion equation of wing/external storage system with high aspect ratio is established by using the medium deformation beam theory and quasi steady aerodynamic model. Considering the nonlinear effects caused by the combined action of aerodynamic load, external load and gravity, the structural nonlinear static deformation of wing / external storage coupling system is solved, and the geometric nonlinear stiffness matrix related to the displacement of element nodes is calculated, Analyze the structural modal frequency and vibration mode characteristics of the wing under linear and nonlinear conditions, study the influence of geometric nonlinear deformation of the wing/multi external storage coupling system on the structural modal and flutter characteristics under forward flight state and different angles of attack, and reveal the internal influence law of the geometric nonlinear effect of the wing structure on its own structural modal and flutter characteristics.

\section{Theoretical Model}

For the dynamic model of high aspect ratio wing / multi external storage coupling system, it is necessary to focus on the geometric nonlinear effect caused by large deformation of high aspect ratio flexible wing structure and the structural modal characteristics under the action of multi-point mass and inertia.

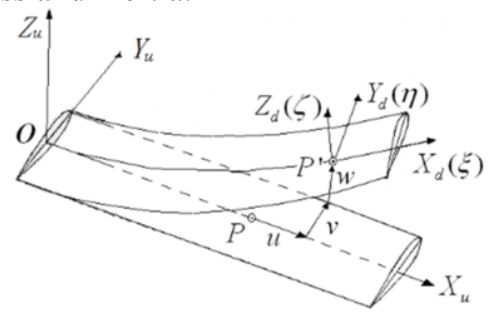

Figure 1. Schematic diagram of wing deformation 
As shown in figure 1 , a point on the elastic axis of the undeformed wing moves to the point $p^{\prime}$ after displacement $(u, v, w)$, and then the wing cross-section containing the point $p^{\prime}$ rotates by an angle $\theta_{1}\left(\theta_{1}=\theta_{0}+\phi\right)$ around the deformed elastic axis, where, $\theta_{0}$ represents the wing pre torsion angle, which $\phi$ is the wing torsion caused by elastic deformation. Considering the small angle assumption, the transformation relationship between the undeformed wing coordinate system and the deformed wing coordinate system is:

$$
T_{d u}=\left(\begin{array}{ccc}
1-\frac{v^{\prime 2}}{2}-\frac{w^{\prime 2}}{2} & v^{\prime} & w^{\prime} \\
-v^{\prime} \cos \theta_{1}-w^{\prime} \sin \theta_{1} & -v^{\prime} w^{\prime} \sin \theta_{1}+\left(1-\frac{v^{\prime 2}}{2}\right) \cos \theta_{1} & \left(1-\frac{w^{\prime 2}}{2}\right) \sin \theta_{1} \\
-w^{\prime} \cos \theta_{1}+v^{\prime} \sin \theta_{1} & -v^{\prime} w^{\prime} \cos \theta_{1}-\left(1-\frac{v^{\prime 2}}{2}\right) \sin \theta_{1} & \left(1-\frac{w^{\prime 2}}{2}\right) \cos \theta_{1}
\end{array}\right)
$$

The position vector of any point on the wing can be expressed as:

$$
r=\{x+u e, v, w\}+\{0, \eta, \zeta\} \cdot T_{d u}
$$

Where, $\{0, \eta, \zeta\}$ is the position vector of any point in the section in the deformed wing coordinate system. The velocity of the point depends on the derivative of the position vector of the point with respect to time, while the kinetic energy of the wing depends on its own velocity. Its expression and corresponding variation are as follows:

$$
\begin{aligned}
& T_{b}=\frac{1}{2} \int_{0}^{R} \iint_{A} \rho_{s} \boldsymbol{V} \cdot \boldsymbol{V} d \eta d \zeta d x \\
& \delta T_{b}=\int_{0}^{R} \iint_{A} \rho_{s} \boldsymbol{V} \cdot \delta \boldsymbol{V} d \eta d \zeta d x
\end{aligned}
$$

In this paper, the elastic wing model is established by using the medium deformation beam theory, that is, assuming that the wing is an anisotropic beam, which produces medium deformation and small strain. The relationship between the stress and strain of the wing and its material elastic modulus constant is as follows:

$$
\left\{\begin{array}{c}
\sigma_{x x} \\
\sigma_{x \zeta} \\
\sigma_{x \eta}
\end{array}\right\}=\left\{\begin{array}{lll}
\mathbf{Q}_{11} & \mathbf{Q}_{15} & \mathbf{Q}_{16} \\
\mathbf{Q}_{15} & \mathbf{Q}_{55} & \mathbf{Q}_{56} \\
\mathbf{Q}_{16} & \mathbf{Q}_{56} & \mathbf{Q}_{66}
\end{array}\right\}\left\{\begin{array}{c}
\varepsilon_{x x} \\
\varepsilon_{x \zeta} \\
\varepsilon_{x \eta}
\end{array}\right\}
$$

After integration along the section and span of the wing, the deformation energy of the wing can be obtained. If the variation of the deformation energy is obtained, the 
corresponding virtual deformation energy can be obtained, and its expressions are as follows:

$$
\begin{aligned}
& U_{b}=\frac{1}{2} \int_{0}^{R} \iint_{A}\left(\sigma_{x x} \varepsilon_{x x}+\sigma_{x \zeta} \varepsilon_{x \zeta}+\sigma_{x \eta} \varepsilon_{x \eta}\right) d \eta d \zeta d x \\
& \delta U_{b}=\int_{0}^{R} \iint_{A}\left(\sigma_{x x} \delta \varepsilon_{x x}+\sigma_{x \zeta} \delta \varepsilon_{x \zeta}+\sigma_{x \eta} \delta \varepsilon_{x \eta}\right) d \eta d \zeta d x
\end{aligned}
$$

In this paper, the quasi steady aerodynamic model is used to describe the aerodynamic load of the wing section. The quasi steady aerodynamic model adopts the lift line theory. As shown in figure 2, the aerodynamic action point is at the quarter chord length, and the aerodynamic load on the airfoil is calculated by the air velocity at the third quarter chord length.

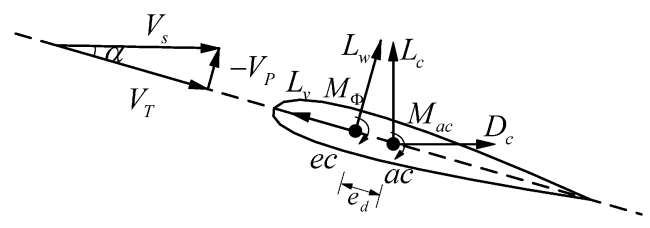

Figure 2. Schematic diagram of aerodynamic load of wing section

Under the deformed wing coordinate system, the aerodynamic lift, drag and moment of the wing section are respectively:

$$
\begin{aligned}
& L_{c}=\frac{1}{2} \rho_{a} V_{s}^{2} c C_{l} \\
& D_{c}=\frac{1}{2} \rho_{a} V_{s}^{2} c C_{d} \\
& M_{a c}=\frac{1}{2} \rho_{a} V_{s}^{2} c^{2} C_{m}
\end{aligned}
$$

Where, $\rho_{a}$ is the air density, $\mathrm{c}$ is the chord length of the wing, $C_{l} 、 C_{d}$ and $C_{m}$ are the aerodynamic lift coefficient, drag coefficient and torque coefficient of the airfoil respectively, which have the following relationship:

$$
\begin{aligned}
& C_{l}=c_{0}+c_{1} \alpha \\
& C_{d}=d_{0}+d_{1}|\alpha|+d_{2} \alpha^{2} \\
& C_{m}=f_{0}+f_{1} \alpha
\end{aligned}
$$

The lift $L_{c}$, resistance $D_{c}$ and moment $M_{a c}$ can be converted to the elastic center EC of the section to obtain the aerodynamic load in the coordinate system after blade deformation: 


$$
\begin{aligned}
& L_{w}=L_{c} \cos \alpha+D_{c} \sin \alpha \\
& L_{v}=L_{c} \sin \alpha-D_{c} \cos \alpha \\
& M_{\Phi}=M_{a c}-e_{d} L_{w}
\end{aligned}
$$

According to the above aerodynamic formula, the aerodynamic virtual work on a single blade can be obtained:

$$
\delta W_{b}=\int_{0}^{R}\left(L_{u}^{A} \delta u+L_{v}^{A} \delta v+L_{w}^{A} \delta w+L_{\phi}^{A} \delta \phi\right) d x
$$

After the finite element discretization of the wing, its energy expression is:

$$
\delta \Pi=\sum_{i=1}^{N}\left(\delta U_{i}-\delta T_{i}-\delta W_{i}\right)
$$

Where $\mathrm{N}$ is the number of elements divided by the wing. By substituting the above expressions of virtual kinetic energy, virtual strain energy and aerodynamic virtual work into the above equations, the aeroelastic motion equation of the wing can be obtained. During the calculation of wing dynamic characteristics, the stiffness matrix of wing structure consists of two parts, one is elastic stiffness matrix $K_{0}$, and the other is nonlinear stiffness matrix $J O K$ considering the node displacement of wing element, namely:

$$
K=K_{0}+J O K
$$

The joint displacement of wing element is caused by the combined action of aerodynamic force and gravity.

Based on the high aspect ratio wing dynamic model established in this paper, the first five natural modal characteristics of Tang Wing model are analyzed. The structural parameters of the wing are shown in table 1,and the calculation results are shown in table 2 .

Table 1. Tang Wing Structural Parameters.

\begin{tabular}{cc}
\hline parameter & value \\
\hline length & $0.4508 \mathrm{~m}$ \\
Torsional stiffness & $0.9539 \mathrm{~N} \cdot \mathrm{m}^{2}$ \\
Swing stiffness & $0.4186 \mathrm{~N} \cdot \mathrm{m}^{2}$ \\
Lead-lag stiffness & $18.44 \mathrm{~N} \cdot \mathrm{m}^{2}$ \\
Moment of inertia per unit & $0.00002056 \mathrm{~N} \cdot \mathrm{m}^{2}$ \\
length & $0.0508 \mathrm{~m}$ \\
Chord length & $0.0417 \mathrm{~kg}$ \\
X-axis moment of inertia & $0.00009753 \mathrm{~N} \cdot \mathrm{m}^{2}$ \\
Y-axis moment of inertia & $0.00003783 \mathrm{~N} \cdot \mathrm{m}^{2}$ \\
Z-axis moment of inertia & $0.00009753 \mathrm{~N} \cdot \mathrm{m}^{2}$ \\
\hline
\end{tabular}


It can be seen from table 2 that the simulation results are basically consistent with the test results, and the maximum error is only $0.32 \%$, which verifies the accuracy of the theoretical model of high aspect ratio wing established in this paper, and can be used to analyze the influence of geometric nonlinear deformation of wing with multi-point external stores on its structural mode.

Table 2. Comparison of natural modes between calculation and test of Tang

\begin{tabular}{cccc}
\hline Mode & Test $/ \mathrm{Hz}$ & Model/Hz & error \\
\hline First order swing & 3.675 & 3.674 & $0.03 \%$ \\
Second order swing & 23.03 & 23.037 & $0.03 \%$ \\
Third order swing & 64.50 & 64.705 & $0.32 \%$ \\
First order lead-lag & 24.39 & 24.387 & $0.01 \%$ \\
First order torsion & 119.5 & 119.628 & $0.11 \%$ \\
\hline
\end{tabular}

\section{Calculation Results}

The dynamic characteristics of high aspect ratio wing / multi plug-in system depend on the influence of wing load deformation. Multiple plug-ins are distributed in series on the wing structure, which is equivalent to the existence of multi-point concentrated mass and inertia. The nonlinear stiffness of the wing structure caused by this load, aerodynamic load and gravity changes the overall stiffness of the structure, Thus, the geometric nonlinear effect of wing large deformation is reflected. In this paper, external stores are arranged at $0.2 \mathrm{~L}, 0.6 \mathrm{~L}$ and $1 \mathrm{~L}$ (L represents the wing span) of Tang Wing, ignoring the influence of external store aerodynamic force on the wing. Firstly, the influence of nonlinear deformation caused by distributed external stores under gravity on the wing structural mode is studied.

Table 3. Comparison of wing structural modes under linear and nonlinear conditions

\begin{tabular}{ccc}
\hline Mode & Linear mode/Hz & Nonlinear mode $/ \mathrm{Hz}$ \\
\hline First order flap & 2.145 & 2.145 \\
Second order flap & 13.43 & 13.43 \\
Third order flap & 41.03 & 41.03 \\
First order lead-lag & 14.07 & 11.25 \\
First order torsion & 18.95 & 19.51 \\
\hline
\end{tabular}




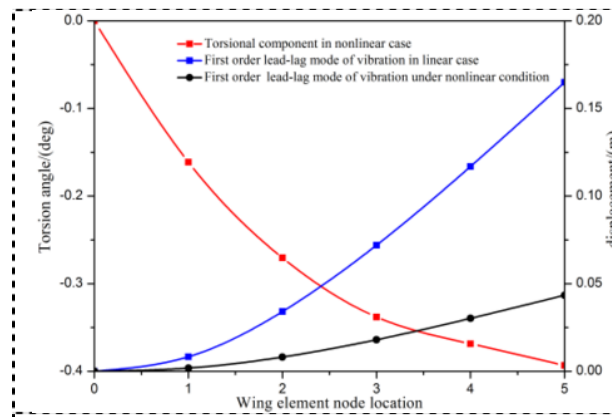

Figure 3. First order mode shape of lead-lag under linear and nonlinear conditions.

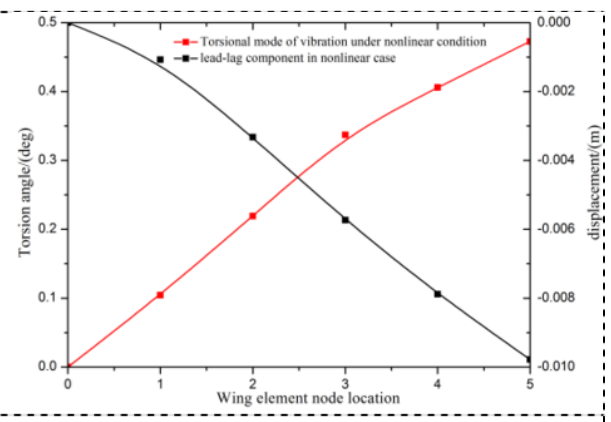

Figure 4. Torsional first-order mode shapes under nonlinear conditions.

It can be seen from table 3 that compared with linear modal analysis, after considering the geometric nonlinear effect, the third-order modal frequency of the wing with three-point plug-in and high aspect ratio is basically unchanged before swing, while the first-order modal frequency of lead-lag decreases and the first-order modal frequency of torsion increases. It can be seen from figure 3 and figure 4 that the first-order mode shape curve of wing lead-lag considering geometric nonlinear deformation decreases significantly, and it is different from the pure lead-lag and pure torsional modes of linear modal analysis. Its mode shape has torsional component, which leads to the coupling of wing lead-lag and torsional motion. Although the torsional first-order mode shape also has lead-lag component, its influence is small. The results show that the geometric nonlinearity changes the modal frequency and vibration mode of the wing structure, which can not be ignored.

In the forward flight state, the aerodynamic load causes large deformation of the flexible wing structure with large aspect ratio, resulting in significant geometric nonlinear effect. Therefore, considering the combined action of aerodynamic load and gravity, the dynamic characteristics of the wing / multi external storage system with large aspect ratio caused by geometric nonlinear deformation are studied.

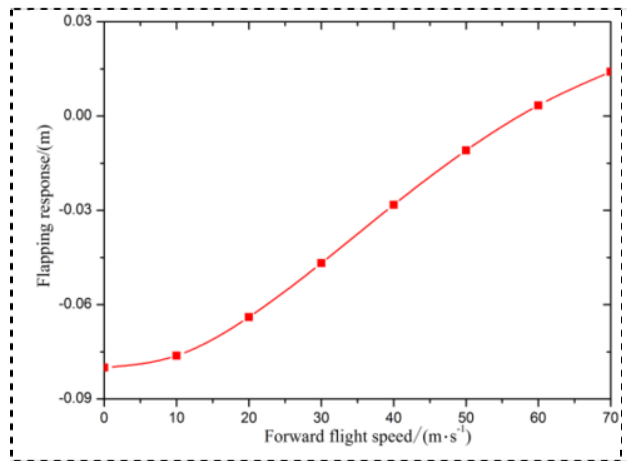

Figure 5. Wing tip flapping response at different forward speeds

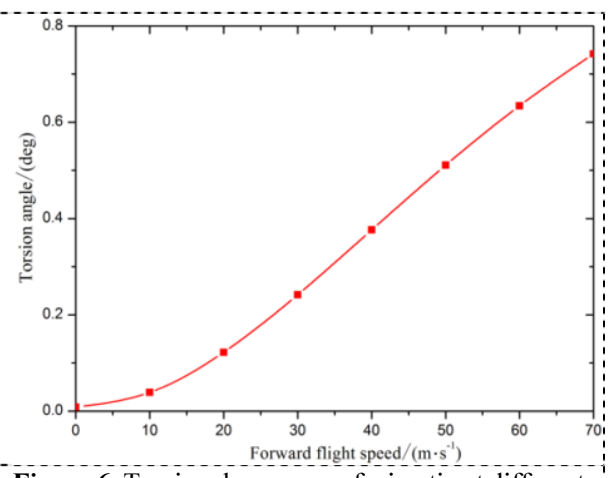

Figure 6. Torsional response of wing tip at different forward speeds 


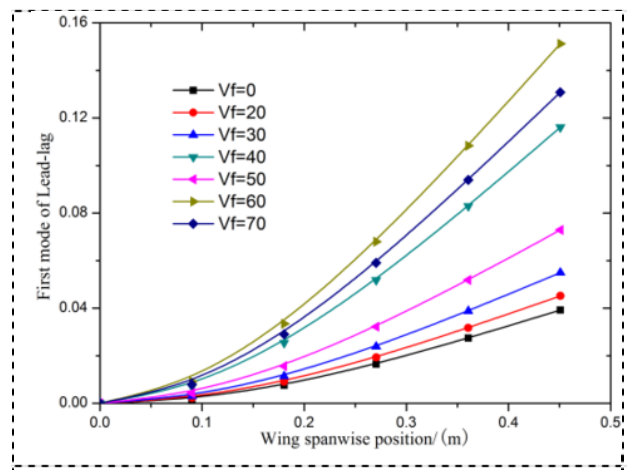

Figure 7. First order modes shape of wing leadlead-lag at different forward speeds

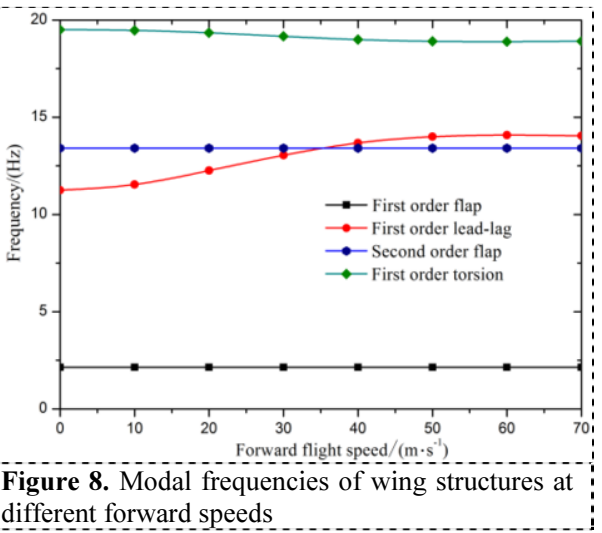

As can be seen from figures 5 to 8 that the wing produces vertical bending deformation and torsional deformation to varying degrees with the increase of forward flight speed. When the current flight speed reaches $60 \mathrm{~m} / \mathrm{s}$, the wing gradually changes from vertical downward bending deformation to horizontal state, that is, the displacement generated by aerodynamic force and gravity just offset. Accordingly, the first-order modal frequency of lead-lag increases from $11.54 \mathrm{~Hz}$ to $14.08 \mathrm{~Hz}$, and the firstorder torsional modal frequency slowly decreases from $19.47 \mathrm{~Hz}$ to $18.89 \mathrm{~Hz}$; When the current flight speed is higher than $60 \mathrm{~m} / \mathrm{s}$, the increase of aerodynamic load causes the wing to gradually change from horizontal state to vertical upward bending deformation. At this time, the first-order modal frequencies of wing lead-lag and torsion slowly decrease or increase. During the change of wing vertical bending state, the amplitude of the first-order mode of wing lead-lag first increases and then decreases. This result shows that the influence of geometric nonlinear effect of wing with high aspect ratio in forward flight is mainly reflected in the first-order mode of wing lead-lag.

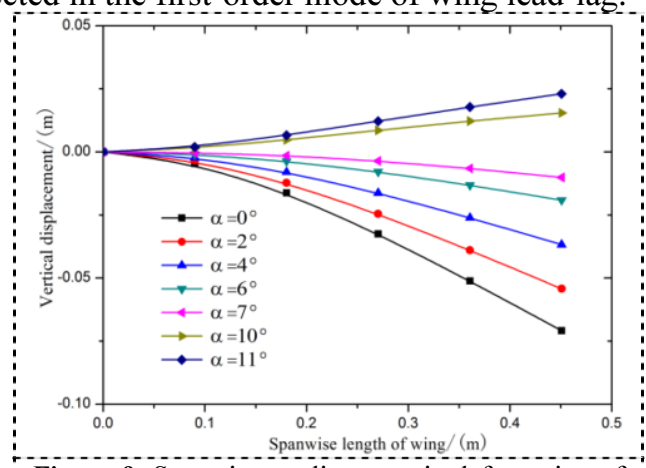

Figure 9. Spanwise nonlinear static deformation of wing at different angles of attack.

It can be seen from the above research that the nonlinear effect caused by the vertical bending deformation of the wing under the influence of lift load will cause the change of the wing structural mode, and the wing angle of attack is one of the main factors generating lift. Therefore, the stall characteristics of the wing with high aspect ratio are not considered, The aeroelastic response and structural characteristics of the wing caused by geometric nonlinearity in the range of small angle of attack are studied. 


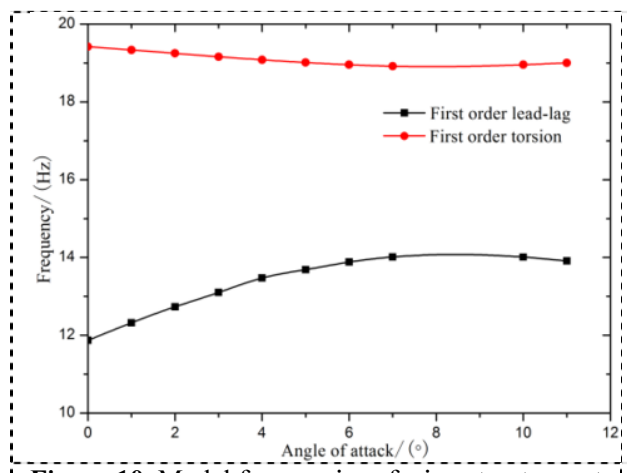

Figure 10. Modal frequencies of wing structures at different angles of attack.

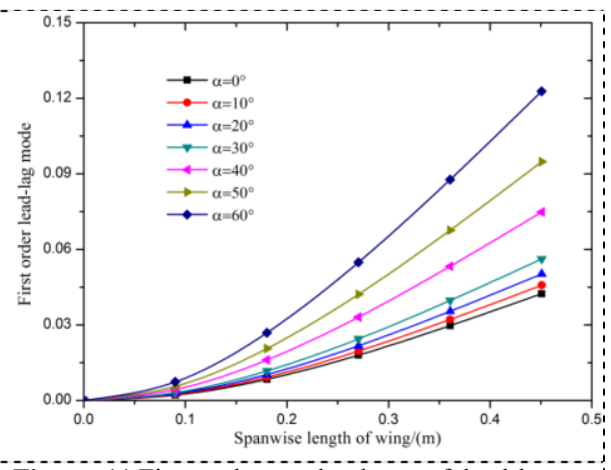

Figure 11.First order mode shape of lead-lag at different angles of attack.

It can be seen from figure 9 that when the incoming flow velocity is constant, the aerodynamic load gradually increases with the increase of the angle of attack, resulting in the evolution of the wing from vertical downward bending deformation to vertical upward bending deformation, and the wing vertical bending amplitude is strong within the range of $0^{\circ}$ to $6^{\circ}$ angle of attack, with strong geometric nonlinear effect. Through the nonlinear modal analysis method, The first-order modal frequencies of lead-lag and torsion at each static equilibrium position are further obtained, as shown in figure 10 .

It can be seen from figure 10 that the change trend of the second-order modal frequency in the figure that when the angle of attack increases from $0^{\circ}$ to $6^{\circ}$, the degree of wing vertical bending decreases greatly, resulting in the increase of the first-order modal frequency of lead-lag from $11.87 \mathrm{~Hz}$ to $14.01 \mathrm{~Hz}$, the slow decrease of the firstorder torsional modal frequency from $19.41 \mathrm{~Hz}$ to $18.91 \mathrm{~Hz}$, and the weakening of geometric nonlinear effect causes the change trend of the first-order modal frequency of lead-lag to slow down gradually, especially near the zero equilibrium point, The firstorder modal frequency of lead-lag and torsion changes very slowly. It can be seen from figure 11 that the amplitude of the first-order vibration mode of wing lead-lag gradually increases with the increase of angle of attack, and the influence of this result on flutter characteristics can not be ignored.

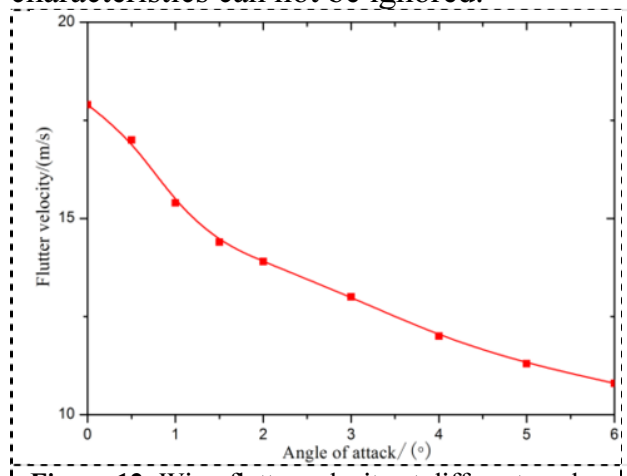

Figure 12. Wing flutter velocity at different angles of attack.

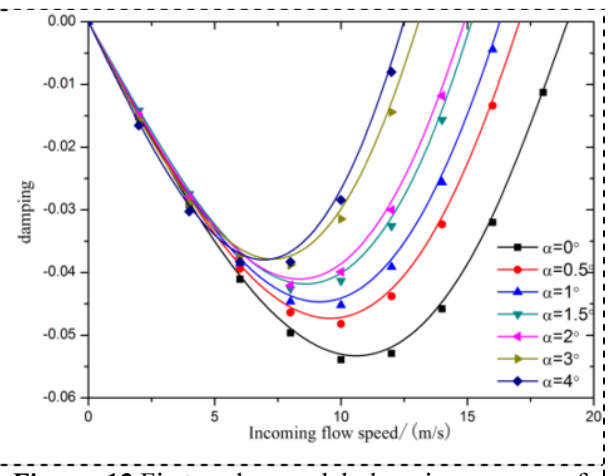

Figure 13. First order modal damping curves of wing lead-lag at different angles of attack

Based on the above nonlinear structural modal analysis of the wing, the flutter characteristics of the wing / multi external storage system with high aspect ratio at different angles of attack are calculated by using the nonlinear flutter analysis method. It can be seen from figure 12 that the variation degree of the wing flutter velocity at 
different angles of attack is basically the same as that of the first-order modal frequencies of lead-lag and torsion, showing a gradual slowing down trend in the range of $0^{\circ}$ to $2^{\circ}$, The large vertical bending of the wing makes the geometric nonlinear effect strong. The first-order modal frequency of lead-lag increases by $7.9 \%$, resulting in the change of flutter velocity of $22.3 \%$. In the range of $2^{\circ}$ to $6^{\circ}$ angle of attack, the change of wing structural vibration characteristics is reduced due to the mitigation of geometric nonlinearity, and the change of flutter velocity is about $21 \%$, The results show that the change of structural vibration characteristics caused by geometric nonlinearity has a direct impact on flutter velocity. It can be seen from figure 13 that under the influence of geometric nonlinear effect, the flutter mechanism of wing / external storage system is bending torsional coupling flutter dominated by the first-order mode of wing lead-lag, and the damping of the first-order mode of wing lead-lag changes significantly at different angles of attack. Considering the variation law of nonlinear vibration characteristics and flutter characteristics caused by the geometric nonlinear effect of large deformation of wing structure, from the perspective of dynamic design, reducing the effect of geometric nonlinear effect will help to improve the aeroelastic stability of wing / multi external storage system to a certain extent.

\section{Conclusions}

In this paper, the aeroelastic motion equation of high aspect ratio wing is established by using the medium deformation beam theory and quasi steady aerodynamic model. Taking Tang Wing as an example, the effects of geometric nonlinear deformation of wing/multi external storage system on the vibration characteristics and flutter characteristics of wing/multi external storage system under forward flight state and different angles of attack are studied. The conclusions are as follows:

1) Compared with linear modal analysis, the geometric nonlinear effect caused by large wing deformation is mainly reflected in the first-order mode of lead-lag, and the first-order mode shape of lead-lag has torsional component, resulting in the coupling between lead-lag and torsional motion;

2) With the increase of forward flight speed or angle of attack, the wing changes from vertical downward bending state to horizontal state under the joint action of aerodynamic load and gravity. The weakening of geometric nonlinear effect causes the first-order modal frequencies of wing lead-lag and torsion to slow down gradually. When the wing is in vertical upward bending deformation state, the second-order modal frequencies decrease or increase accordingly. In this process, The amplitude of the first mode of wing lead-lag increases first and then decreases;

3) Under the action of geometric nonlinearity, the wing flutter mechanism is the bending torsional coupling flutter dominated by the first-order mode of lead-lag, which is directly affected by the change of wing structural vibration characteristics. By reducing the influence of geometric nonlinearity of the wing, it has a certain positive effect on the wing flutter speed. 


\section{References}

[1] Ehernberger L J, Casey Donohue. A review of solar powered aircraft flight activity[R]. NASA Report, 2004.

[2] WANG Wei. Research on nonlinear aeroelasticity and flight mechanics of solar UAV [D]. Doctoral dissertation, Xi'an: Northwest University of technology. 2011.

[3] Dowell E,Edwards J,Strganac T. Nonlinear Aeroelasticity[J].Journal of Aircraft,2003,40:857-874.

[4] Weihua Su,Carlos E,Cesnik S. Nonlinear Aeroelasticity of a very flexible blended-wing-body Aircraft[J].Journal of Aircraft,2010,47:1539-1553.

[5] YANG Zhi-chun, DANG Hui-xue, LY Yi. Numerical simulation of nonlinear aeroelastic characteristics of high aspect ratio wing [C] , the 11th National aeroelastic academic exchange, Kunming: 2009.

[6] E.H. Dowell, D.M. Tang. Nonlinear aeroelasticity and unsteady aerodynamics[R].AIAA-2002-0003

[7] LENG Jia-zhen, XIE Chang-chuan, YANG Chao. Effect of horizontal bending stiffness on flutter of high aspect ratio wing [J]. Journal of Beijing University of Aeronautics and Astronautics, 2009, 35 (6): 718 722 .

[8] Librescu L,Song O.Dynamic of composite aircraft wings carrying external stores[J].AIAA Journal,2008,46(3):568-572

[9] Mayuresh J P,Dewey H H.Nonlinear Aeroelasticity and Flight Dynamics of High-Altitude LongEndurance Aircraft[J].Journal of Aircraft,2001,38:88-94

[10] AN Xiao-min, XU min. a nonlinear aeroelastic solution method under large geometric deformation [J]. Journal of mechanics, 2011, 43 (1): 97-104.

[11] WANG Wei, DUAN Zhuo-yi, GENG Jian-zhong. Geometric nonlinear structural modeling of large flexible wing based on Cr theory [J]. Acta Aeronautica Sinica, 2017,38 (S1): 721544.

[12] XIE Chang-chuan, YANG Chao. Linearization method of nonlinear aeroelastic stability for complete aircraft with high-aspect-ratio wings [J]. Science China Series E-Technology Science, 2011, 54: 403411.

[13] Mayuresh J P,Dewey H H.Nonlinear Aeroelasticity and Flight Dynamics of High-Altitude LongEndurance Aircraft[J].Journal of Aircraft,2001,38:88-94.

[14] HU Zhi-yong, YOU Wu, ZHANG Jian.Geometrically nonlinear aeroelastic analysis of solar UAV wing [J]. Journal of Air Force Engineering University, 2019,20 (2): 1-6. 